\title{
Virtual Screening based prediction of potential drugs for COVID-19
}

\author{
Dr. Talluri Sekhar \\ Professor, Department of Biotechnology, GITAM \\ Visakhapatnam, India
}

\section{Abstract}

SARS-CoV-2 is the betacoronavirus responsible for the COVID-19 pandemic. It was listed as a potential global health threat by WHO due to high mortality, high basic reproduction number and lack of clinically approved drugs and vaccines for COVID-19. The genomic sequence of the virus responsible for COVID-19, as well as the experimentally determined three dimensional structure of the Main protease (Mpro) are available. The reported structure of the target Mpro was utilized in this study to identify potential drugs for COVID-19 using molecular docking based virtual screening of all approved drugs. The results of this study confirm preliminary reports that some of the drugs approved for treatment of other viral infections have the potential for treatment of COVID-19. Selected antiviral drugs, approved for human therapeutic applications, were ranked for potential effectiveness against COVID-19, based on predicted binding energy to the target Mpro of SARS-CoV-2, and novel candidates for drug repurposing were identified in this study. In addition, potential mechanisms for beneficial off target effects of some drugs in clinical trials were identified by using molecular docking.

\section{Keywords}

Virtual Screening, Molecular Docking, Drug Repurposing, Drug Repositioning, Anti-Viral drugs, Coronavirus, COVID-19, 2019-nCoV, SARS-CoV-2

\section{Abbreviations}

Direct Acting Antivirals (DAA), Corona Virus Disease-2019 (COVID-19), Severe Acute Respiratory distress Syndrome Corona Virus-2 (SARS-CoV-2) 


\section{Introduction}

COVID-19 is a highly infectious disease associated with high mortality [1, 2], and there are no approved drugs or vaccines for this disease, although chloroquine and hydroxychlroquine have been authorized for emergency use [3]. World Health Organization has declared COVID-19 to be a public health emergency of international concern [4]. SARS-CoV-2, the betacoronavirus responsible for COVID-19, is a positive single-stranded RNA virus belonging to the coronoviridae family [1, 5]. The previous name for this virus was 2019-nCoV [6]. The genome of SARS-CoV-2 has been sequenced [7]. The availability of the genome sequence and the subsequent phylogenetic studies revealed that the genomic sequence of SARS-CoV-2 has $96 \%$ identity to the bat-coronavirus and 79.6\% sequence identity to SARS-CoV [8]. Although there are no approved drugs or vaccines for COVID-19, a number of clinical trials are in progress $[3,9]$ and a variety of potential therapeutic strategies are under investigation $[10,11]$.

Computational methods can be utilized for design and engineering of drugs [12, 13, 14, 15]. The low time requirements of computational methods are conducive for high throughput screening of available drugs to identify potential drugs for novel diseases as well as to predict the adverse effects of novel drugs $[16,17]$. Development of novel drugs is a time consuming process and generally several years of work are required for clinical approval [18]. Drug repositioning, also known as repurposing, is an effective strategy to combat novel diseases caused by infectious agents that spread rapidly [19, 20]. Drugs that have been approved for some disease, are safe for human use at approved dosage levels [21]. However, clinical trials are necessary to ensure that such treatment is better than a placebo [22].

Several anti-viral agents exhibit in-vitro and in-vivo anti-SARS-CoV-2 activity and have potential for drug repurposing, e.g., Hydroxychloroquine, Chloroquine, Remdesivir, Favipiravir, Nitazoxanide [23, 24, 25]. It has been reported that Chloroquine phosphate shows anti COVID-19 activity in clinical studies [26]. A small clinical trial has concluded that Hydroxychloroquine and Azithromycin are effective for treatment of COVID-19 [27]. Large scale clinical trials are under progress and additional clinical trials are being planned to confirm the effectiveness of some proposed drugs [28].

The similarity of SARS-CoV-2 to other coronaviruses enabled rapid identification of targets for drug design because of substantial earlier work on development of drugs for other coronaviruses such as SARS-CoV and MERS-CoV [29]. The main protease of SARS-CoV-2 has been identified as an attractive target for drug design and development because it plays an essential role in viral replication [30]. It is a Cys-His protease and its Cysteine can be targeted by covalent drugs [31]. The viral RNA is expressed as a polyprotein and the main protease of SARS-CoV-2 is required for cleavage of this polyprotein at multiple cleavage sites [32]. The main protease of SARS-CoV-2 has a high degree of specificity and yet its specificity does not match that of human proteases [33]. Therefore, we may expect that it should be possible to design or identify inhibitors of this viral protease that do not inhibit essential human protease activity.

Several clinical trials are assessing the potential of protease inhibitors approved for treatment of other viral infections, such as Lopinavir and Ritonavir. Lopinavir and Ritonavir were used in 
clinical studies for treatment of COVID-19 [34, 35]. Lopinavir and Ritonavir target the main protease (Mpro) of SARS coronavirus [36]. Several studies have utilized homology based models of SARS-CoV-2 Mpro to screen libraries of compounds for prediction of potential drugs for COVID-19 [37]. However, the predictions of virtual screening studies and binding energy calculations are generally more accurate if a high resolution experimental structure of the target is available [38]. Therefore, the recently available experimental structures of the Main protease, Mpro of SARS-CoV-2 [39], were utilized in the current study as the target for molecular docking based virtual screening. Large scale docking studies for rapid identification of potential inhibitors of SARS-CoV-2 Mpro have been reported earlier [40, 41]. However, in the study reported here, the focus is on repurposing of drugs approved for clinical applications. In addition, in the studies reported here, high resolution information regarding the target structures was utilized to investigate the effects of binding site flexibility on the predicted binding affinities. The predictions of this study will provide information that can be utilized for choice of candidate drugs for in vitro studies, in vivo studies and clinical trials and to obtain mechanistic insights regarding the results of such experimental studies. 


\section{Methods}

\subsection{Target preparation}

The structures of the target protein SARS-CoV-2 Mpro were obtained from the Research Collaboratory for Structural Bioinformatics Protein Data Bank (RCSB PDB) [42]. The first structure of the target with a covalent inhibitor, N3, was available from $5^{\text {th }}$ February, 2020 in the RCSB PDB and the second version of this structure, 6LU7 (2.16 ̊ Resolution) [39, 43], was utilized in this study for screening of SuperDRUG2 database. A newer version of the complex with N3, with

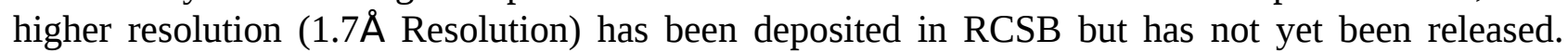
Higher resolution structures of the target complex with other ligands, as well as in the apo form are also available [40]. The target structure having highest resolution for Apo form (6YB7, 1.25 Resolution) and the target structure having highest resolution with a bound ligand (5R82, 1.31 Resolution) were selected for docking studies. Hydrogens for $\mathrm{pH} 7.0$ and charges were added to the protein and a pdbqt format file was generated by using Open Babel (Obabel) [44] . Autodock Vina requires receptor structure in pdbqt format. SMINA can utilize input receptor files in both pdb and pdbqt formats. Avogadro [45], Pymol (DeLano, 2002) and Rasmol [46] were used for visualization.

\subsection{Target active site analysis}

Active site residues were identified by their proximity to the N3 ligand in 6lu7 by using Rasmol. Pymol and TMalign [47] were used for superposition of the target structures in 6lu7, 5r82 and 6yb7. Custom scripts and LibreOffice were used for calculation of residue wise RMSD.

\subsection{Ligand preparation}

The list and structures of approved drugs were obtained from SuperDRUG2 database. SuperDRUG2 maintains a database of drugs approved for clinical use [48, 49]. The version of the database downloaded on 18 February, 2020 contained 3639 approved drugs. For the purpose of this study, the term 'approved drug' is used for drugs in this list. For each molecule in the 3D conformer list obtained from SuperDrug2, the first conformer was utilized in this study. Gasteiger charges were added, and structural optimization was carried out with MMFF94 forcefield using steepest descents followed by conjugate gradient minimization. Subsequently, the structures were converted into pdbqt format, for use in docking calculations with Vina. These structures were further converted to mol2 format for calculations with SMINA. Avogadro and Pymol were utilized for visualization of the ligands.

\subsection{Clinical Trials Data}

Data related to Clinical Trials were downloaded from clinicaltrials.gov on $6^{\text {th }}$ March, 2020 and WHO clinical trials index on $3^{\text {rd }}$ April, 2020. Drug like small molecules in interventional category were selected for drugs in Phase 1,2,3 and 4 clinical trials. Clinical trials listed with unspecified Phase or Phase 0 were not included in this study. Some of these investigational molecules in these clinical trials have been approved for other indications, whereas others are not. The investigational molecules that were approved for other indications were listed in SuperDRUG2, whereas others were downloaded from Pubchem [50] or Drugbank [51] or ZINC 15 [52]. Low energy 
conformations were generated and then hydrogens and gasteiger charges were added using Obabel. Obabel was used for geometry optimization with MMFF94 forcefield. Computational studies were not undertaken for Carrimycin, currently in Phase 4 of clinical trials, due to lack of structural data.

\subsection{Docking}

Autodock Vina [53] and SMINA [54] were utilized for molecular docking studies, because they are freely available and the scores and ranking of these tools have been validated in independent studies [55]. Virtual Screening workflow was implemented using Obabel, Autodock Vina, SMINA and customized Python and shell scripts. Pymol and Rasmol were utilized for visualization of the docked results.

Autodock Vina was used for docking calculations that did not involve flexible active sites residues (rigid receptor docking). The exhaustiveness parameter that controls the extent of the search was chosen as 8, and 9 modes were generated for each ligand.

SMINA, a derivative of Vina, was used for docking calculations using both rigid and flexible active site residues. The flex_hydrogens option was used in SMINA to enable comparability of Vina and SMINA results. The same set of parameters were utilized for Vina and SMINA, with the following exceptions. The exhaustiveness parameter was set to 16 for docking calculations involving flexible residues in the active site. Active site residues H41 and C145 were chosen for flexible docking with SMINA because these two residues are highly conserved. In addition, residues M49 and Q189 were chosen for SMINA flexible docking studies because these two residues had the highest RMSD among the active site residues, when residues 1-195 of 6lu7 and 5r82, comprising the active site domains of the two complexes of SARS-CoV-2 Mpro, were superposed.

Dissociation constants were calculated from Vina scores with 6lu7 as the target for docking partly because the structure of the N3 ligand in 6lu7 has a higher degree of structural similarity to the most promising candidate drugs. SMINA predicted binding affinities are higher in most cases because of the use of flexible residues at the active site. Hence the rigid body docking scores for Vina are conservative estimates for binding affinity. 


\section{Results}

The inhibitor N3 was bound to Mpro in the structure of the complex (6lu7.pdb) determined from Xray crystallographic data. The binding energy and binding modes of N3 were predicted with Autodock Vina and SMINA to validate the docking protocol used in this study. The lowest energy binding mode predicted by Vina and the lowest energy binding mode predicted by SMINA were found to match the experimental binding mode of the inhibitor N3. However, there were minor differences in the binding energies and binding modes determined by the two methods, due to small differences in conformational search algorithms and inherent randomness of conformational search. The superposition of the predicted binding modes and the experimental results are displayed in Figure 1. These two methods may be deemed to yield similar results for the purpose of this study.

The energies of the first two binding modes of N3 reported by Vina were identical $(-8.1 \mathrm{kcal} / \mathrm{mol})$, but the first one was significantly different from the native binding mode (conformational rmsd of $4.8 \AA$ for non-hydrogen atoms), while the second had an excellent match with the native binding mode (conformational rmsd of $2.2 \AA$ for non-hydrogen atoms; Figure 1a). The conformation of the configuration ranked fourth by Vina $(-7.8 \mathrm{kcal} / \mathrm{mol})$ was closest to the experimental structure (conformational rmsd of $1.6 \AA$ for non-hydrogen atoms).

The configuration ranked first by SMINA (-8.1 kcal $/ \mathrm{mol})$ exhibited a better match to the experimentally determined binding conformation of the inhibitor N3 (conformational rmsd of 1.9 $\AA$ for non-hydrogen atoms; Figure 1b) than the lowest energy configuration reported by Vina (conformational rmsd of $2.2 \AA$ for non-hydrogen atoms). However, the SMINA configuration with the best match to the experimental binding conformation (conformational rmsd of $1.7 \AA$ for nonhydrogen atoms) corresponded to weaker binding $(-7.3 \mathrm{kcal} / \mathrm{mol})$. Such minor differences are not unexpected, since these docking studies did not consider the covalent bonding between the ligand and the receptor which was present in the experimental structure.

The list of approved drugs listed in SuperDrug2 database was used to identify drugs that have the potential to bind to Mpro of SARS-CoV-2 by using Vina. For most drugs the difference between the binding energy calculated with Vina and with flexible docking with SMINA were less than 0.5 $\mathrm{kcal} / \mathrm{mol}$ for the target structure 6lu7. The drug with the best binding in the database was Beclabuvir, with a predicted binding energy of $-10.4 \mathrm{kcal} / \mathrm{mol}$ (Vina) and a predicted binding energy of $-10.0 \mathrm{kcal} / \mathrm{mol}$ for flexible docking with SMINA for the target structure 6lu7. Although the binding mode of Beclabuvir is similar to that of N3 in the experimentally determined structure of the complex of SARS-CoV-2 Mpro and N3, there are some significant differences in the sites of interaction (Figure 2). 56 close contacts $(<3 \AA$ ) were observed between the bound conformation of Beclabuvir and the target, at subsite S1' (T25), subsite S1 (L141, N142, E166), subsite S2 (H41, M49, Y54, M165, D187), subsite S4 (Q189) and residues P52, G143, H164, R188 and T190. The key differences are that Beclabuvir has fewer interactions with the residues at the hydrophobic S4 subsite than the N3 ligand in the experimental structure of the complex.

Saquinavir, an antiviral protease inhibitor, was predicted to have a binding energy of $-9.3 \mathrm{kcal} / \mathrm{mol}$ with Vina and $-9.2 \mathrm{kcal} / \mathrm{mol}$ with SMINA for the target structure 6lu7. The predicted binding mode of Saquinavir displays a high degree of similarity to that of the ligand N3 in the experimentally determined structure of the complex of SARS-CoV-2 Mpro and N3 (Figure 3). 52 close contacts $(<3 \AA$ ) were observed between the bound conformation of Saquinavir and the target, at subsite S1' (T25), subsite S1 (F140, L141, N142, H163, E166), subsite S2 (H41, M49, M165, D187), subsite S4 (L167, Q189) and residues T26, G143, S144, P168, R188, T190 and A191. Therefore, the predicted binding of Saquinavir is very similar to the observed binding of the N3 ligand. 
The predicted binding energies for selected antiviral drugs that have potential for therapy of COVID-19 are displayed in Table 1. The docking scores are sensitive to the choice of target structure as well as the choice of the residue designated as flexible. The maximum difference between the scores is $2.4 \mathrm{kcal} / \mathrm{mol}$, with a mean difference of $1.1 \mathrm{kcal} / \mathrm{mol}$ between the best score and the worst score, for the drugs in Table 1. The largest differences were observed for Beclabuvir, Darunavir and Ritonavir. The better scores for SMINA are likely to be due to the increased flexibility in the active site, which may permit certain binding modes that are forbidden in the rigid model of the target used in these Vina calculations. However, there are substantial differences in the structure of the Apo form of SARS-CoV-2 Mpro obtained from X-ray diffraction data and the structures of SARS-CoV-2 Mpro-ligand complexes obtained from X-ray diffraction data. As a consequence, docking score predicted affinities were much lower for most ligands if the structure of Apo form of SARS-CoV-2 Mpro was used as a target, even after taking into account the flexibility of some of the residues at the active site. According to Table 1, Indinavir is the only drug that has a better score if the structure of the Apo form of SARS-CoV-2 Mpro is used as a target.

The list of highest ranked approved drugs, based on Vina scores, are displayed in Table 2. The corresponding binding energies for flexible docking, estimated by using SMINA are also shown in Table 2. The data in Table 2 indicates that there are many potential inhibitors other than the recognized antiviral protease inhibitors. It is likely that some of these are false positives. However, in vivo results show that Digitoxin and Digoxin, the compounds having the best SMINA scores in this list, are effective inhibitors of SARS-CoV-2 and MERS-CoV in Vero cells with a high therapeutic index (unpublished data, [56, 57]). The reported values of IC50 for SARS-CoV-2, determined from immunofluorescence data, were $0.19 \mu \mathrm{M}$ for Digoxin, and $0.23 \mu \mathrm{M}$ for Digitoxin, which are comparable to the dissociation constants estimated from Vina scores $(-9.2 \mathrm{kcal} / \mathrm{mol}, 0.18$ $\mu \mathrm{M})$. Digoxin is a cardiac glycoside and can be used for treatment of chronic atrial fibrillation, a particular type of cardiac arrythmia. For people with this type of cardiac arrythmia, Digoxin/Digitoxin, may provide a dual function as anti-viral and anti-arrythmic drug. This hypothesis needs to be investigated immediately because a high proportion of patients admitted into hospitals with COVID-19 have cardiac arrythmia [58].

Several small molecule drugs are under investigation in Clinical trials for therapy of COVID-19. The small molecule drugs in Phase 1, 2, 3 or 4 of clinical trials were investigated for potential interactions with SARS-CoV-2 Mpro by using Vina and SMINA. The drugs with highest Vina scores are displayed in Table 3. The Status column of Table 3 indicates the highest phase clinical trial in which the drug of interest was included, either individually or in combination. Many clinical trials involve drug combinations. In addition, the same drug may be under investigation in more than one clinical trial. The SMINA scores reported in Table 3 are the best scores obtained from docking to the receptor structures obtained from 6lu7 (flexible residues 41, 145), 6lu7 (flexible residues 49, 189), 5r82 (flexible residues 49, 189) and 6yb7 (flexible residues 49, 189). The individual SMINA scores reported in Appendix 1 indicate that there is substantial receptor structure based variation in the SMINA scores with an average difference of $1.0 \mathrm{kcal} / \mathrm{mol}$ between the best and worst SMINA score, and a maximum difference of $1.9 \mathrm{kcal} / \mathrm{mol}$. Most of the variation is due to the difference in the SMINA scores for binding to the structure obtained from the Apo form of the receptor. According to Table 3, the difference between the estimated binding energies is less than $1.2 \mathrm{kcal} / \mathrm{mol}$ for binding energies estimated with a rigid receptor (Vina) and for those estimated with limited flexibility at the active site (SMINA), with a mean difference of $0.5 \mathrm{kcal} / \mathrm{mol}$. The highest difference between Vina and SMINA scores was observed for Lopinavir $(1.1 \mathrm{kcal} / \mathrm{mol})$. Although the Vina score for Lopinavir $(-7.4 \mathrm{kcal} / \mathrm{mol})$ corresponding to an estimated dissociation constant of 3.7 $\mu \mathrm{M}$ is consistent with the experimental IC50 for Lopinavir which is also in the single digit micromolar range, its rank compared to other drugs is not very high based on Vina score. Lopinavir's rank improves substantially if SMINA scores for flexible docking are used. The improvement in SMINA scores for Lopinavir is due to the changes in the receptor conformation at 
the active site for residues Met49 and Gln189 (Table 3). The improvement in the SMINA scores ($8.5 \mathrm{kcal} / \mathrm{mol}$ ) is observed for the $2.16 \AA$ structure (6lu7) as well as for the higher resolution $1.31 \AA$ structure (5r82), but not for the Apo form (6yb7, $1.25 \AA$ ). SMINA scores indicate that the interaction of other protease inhibitors is also weaker if the Apo is used as the target structure. These data indicate that movements at the residues Met49 and Gln49, as well as minor movements of other residues at the binding site are important for the interaction of Lopinavir with the main protease of SARS-CoV-2. Conformational flexibility has also been observed in X-ray diffraction based structures of main protease of SARS-CoV [59].

All protease inhibitors listed in clinical trials have predicted dissociation constants that are equal to or better than $4 \mu \mathrm{M}$ and SMINA scores $\leq 8.0 \mathrm{kcal} / \mathrm{mol}$. The four drugs that have the highest SMINA scores are: Danoprevir, Darunavir, Lopinavir and Methylprednisolone. The drugs that have the best SMINA scores also are the ones that have the biggest differences from Vina scores, indicating that changes in receptor conformation play an important role in the binding of these drugs. In addition to the protease inhibitors, four drugs have high predicted binding affinities for the main protease of SARS-CoV-2, Sildenafil, Itraconazole, Sofosbuvir and Methylprednisolone. These may represent false positives or potential for dual roles for these drugs. The large volume of the binding site of SARS-CoV-2 enables binding of molecules which may not inhibit its activity. 


\section{Discussion}

The primary limitations of the docking based virtual screening strategy are the high false positive rates and low correlation coefficients between estimated binding energy and experimental measures of activity reported in some earlier studies [60,61]. Even if the same scoring function and target receptor structure are used, differences in predicted binding constants can arise due to differences in charges assigned to the receptor, the method used for relaxation of the receptor, flexibility of the receptor, charge type added to the ligand, differences in use of united atoms, size and centering of the grid used to calculate the molecular potential, the exhaustiveness of the search as well as due to the randomness of the search procedure [62, 63]. Therefore, it is not surprising that there are differences in the potential drugs identified by molecular docking based virtual screens reported by different groups [64]. Despite these limitations, the estimates of binding energy provide valuable information that can be used to guide and analyze the results of complimentary studies [65, 66], such as, Molecular Dynamics based estimates of free energies, in vitro studies, in vivo studies and clinical studies. The limitations of docking have been mitigated in this study by use of high resolution experimental structures of the target, utilization of precise knowledge of the location of binding site, availability of structural information regarding the Apo as well as complex, and structural information regarding multiple ligands in complex with the target.

Lopinavir, Ritonavir and Nelfinavir have been reported in earlier studies as potential drug candidates that target Mpro of SARS-CoV-2. The results of this virtual high throughput screening study, based on the reported structure of Mpro obtained from X-ray crystallographic data, are consonant with the earlier predictions. The binding energies for Lopinavir and Ritonavir (Vina score of -7.4 to $-7.7 \mathrm{kcal} / \mathrm{mol}$ ), predicted in this study, are consistent with preliminary clinical data indicating effectiveness for these drugs [34]. Potentially more effective drugs, with higher affinity, have been identified in this study. Beclabuvir, with a predicted binding energy of $-10.4 \mathrm{kcal} / \mathrm{mol}$ (Vina), was identified as the drug with the best binding energy (Table 2). However, based on approval status and similarity of binding mode, Saquinavir with a predicted binding energy of -9.3 $\mathrm{kcal} / \mathrm{mol}$ (Vina) is the best novel candidate drug (Table 1 and Figure 3).

Darunavir, an HIV protease inhibitor, is predicted to have the best binding energy $(-7.8 \mathrm{kcal} / \mathrm{mol}$, Vina; $-8.8 \mathrm{kcal} / \mathrm{mol}$ SMINA) among the class of protease inhibitors that have been approved for human clinical indications and are currently in clinical trials for treatment of COVID-19 (Table 3). The Virtual Screening of all drugs approved for clinical use identified Saquinavir, also an HIV protease inhibitor, with a predicted binding energy of $-9.3 \mathrm{kcal} / \mathrm{mol}$ (Vina), indicating a potential for increased efficacy (Table 1).

Many drugs that are under consideration for treatment of COVID-19 were chosen based on the similarity of the protein sequences of SARS-CoV-2 to the drug targets of other coronaviruses [67]. However, for many drugs, similarity of sequence does not guarantee that a drug developed for a similar virus will be effective for the virus of interest. Even a single mutation is sufficient to cause substantial changes in the binding affinity. Docking studies, are more sensitive to changes in the structure of the target and provide a more reliable guide to identify drugs that have the potential to be repurposed for treatment of COVID-19. The results of Molecular Docking for compounds currently in clinical trials predicted that Danoprevir, currently in Phase 4 clinical trials for treatment of COVID-19, has the best binding energy for inhibition of Mpro of SARS-CoV-2 (-9.3 kcal/mol, Vina, Table 3). Danoprevir is an inhibitor of NS3 protease used for treatment of Hepatitis C. Danoprevir (included in Ganovo) has been approved by China's FDA for treatment of Hepatitis C [68]. The screening of all drugs in the approved category list in SuperDRUG2 database identified Beclabuvir, an inhibitor of Hepatitis C NS5B protease, as the drug having the best binding energy (-10.4 kcal/mol, Vina) for interaction with Mpro of SARS-CoV-2, indicating a potential for higher 
efficacy. Beclabuvir has been approved as part of a fixed dose combination in Japan for treatment of Hepatitis C virus genotype 1 [69]. However, at present, neither Beclabuvir nor Danoprevir are listed at the FDA server at fda.gov for list of approved drugs.

Methyprednisolone, currently in Phase 4 clinical trials for therapy of COVID-19, is a glucocorticoid that is approved for disorders that require inhibition of pro-inflammatory signals. Thalidomide, an immunomodulatory and anti-inflammatory agent has been used with Methylprednisolone for successful treatment of one case of COVID-19 (unpublished data, [70]). One of the causes of the COVID-19 associated mortality is that high viral loads cause severe inflammation and the body responds with a cytokine storm [71]. Overreaction of the human immune system to such a cytokine storm can result in multiple organ failures and production of large amounts of mucous in the lungs [72]. The presence of large amount of mucous in the lungs is a major contributor towards the acute respiratory distress syndrome (ARDS). Short term, low to moderate doses of methylprednisolone or equivalent corticosteroids have been recommended for critically ill COVID-19 patients to mitigate these effects [73]. Molecular Docking based binding energy (Table 3) indicates that Methylprednisolone may have an unexpected ability to bind and inhibit Mpro of COVID-19. Surprisingly, Methylprednisolone, whose mode of action is not recognized as protease inhibition, is predicted to be an inhibitor of SARS-CoV-2 Mpro, with a calculated dissociation constant in the $\mu \mathrm{M}$ range (Table 3). Therefore, Methylprednisolone may assist in inhibition of viral replication, in addition to its primary role of immunosuppression. This prediction, if confirmed, indicates that Methylprednisolone has multiple modes of action, and that the off target effects are beneficial for therapeutic applications. The potential advantages of drugs for COVID-19 with antiviral and antiinflammatory actvities have been described earlier [74]. Baricitinib an anti-inflammatory drug for rheumatoid arthritis was identified as a potential antiviral using an information graph approach [74], and it is being investigated in Phase 3 clinical trials for treatment of COVID-19. The data in Table 3 suggests its antiviral activity may involve protease inhibition of SARS-CoV-2 Mpro.

Although the Mpro of SARS-CoV-2 is a cysteine protease and its active site is devoid of the C2 symmetry of the aspartic protease of HIV, the molecular docking based binding energies reported in this study, as well as unpublished reports of binding free energy calculations, predict that several HIV-protease inhibitors that are currently approved for treatment of AIDS, such as Saquinavir, Darunavir, Indinavir, Nelfinavir, Tipranavir, Lopinavir and Ritonavir, have the potential to be repurposed for treatment of COVID-19. In addition, the results of this study indicate that protease inhibitors of Hepatitis C virus, Beclabuvir and Danoprevir, have stronger binding affinity for Mpro of SARS-CoV-2, than the strongest binding HIV-protease inhibitors.

A clinical trial of Lopinavir-Ritonavir for treatment of severe COVID-19 has yielded negative results [35]. Although, it is possible that Lopinavir-Ritonavir may be useful for less severe cases and in combination with other drugs [75], it would be worthwhile to investigate the repurposing of the approved drugs identified in this study which are predicted to have higher affinity for SARSCoV-2 Mpro. 


\section{Conclusions}

Drug repurposing is one of the most attractive options for life threatening pandemics that propagate rapidly, such as COVID-19, especially when there is no alternative medicine or vaccine. Computational studies can be used for rapid identification of potential therapeutics for drug repurposing [76]. The results of this virtual screening predict that the cardiac glycosides, Digoxin and Digitoxin, which are used to manage atrial fibrillation, are inhibitors of the main protease of SARS-CoV-2. Patients with cardiovascular disorders are at higher risk from COVID-19 [77]. Therefore, observational studies on patients using Digoxin may be used to assess the potential utility of these cardiac glycosides for prevention or alleviation of COVID-19.

Saquinavir and Beclabuvir were identified as the best novel protease inhibitor candidates for COVID-19 therapy by using Virtual Screening of clinically approved drugs and the structure of SARS-CoV-2 Mpro determined from X-ray diffraction data. The results of this study also rationalize the limited data regarding effectiveness of drugs for COVID-19 therapy, and provide information that can be utilized for choice of candidate drugs for in vitro studies and in vivo studies. The predicted binding and ranking of drugs will also be useful to interpret the results of ongoing clinical trials that are testing small molecule drugs for effectiveness against COVID-19. 


\section{Tables}

Table 1. Predicted binding energies of selected approved antiviral drugs ( $\mathrm{kcal} / \mathrm{mol})$. The target protease, Mpro of SARS-CoV-2, was rigid for Vina calculations and SMINA calculations were carried out with flexible residues at the active site. The resolution of the target structure is given in brackets $(\AA)$. The ligands (RZS or N3) were removed from the structure of the complex before docking with the ligand of interest.

\begin{tabular}{|l|c|c|c|c|c|}
\hline & Vina & SMINA & SMINA & SMINA & SMINA \\
\cline { 2 - 6 } & $\begin{array}{c}\text { 6lu7 (2.16) } \\
\text { Complex with } \\
\text { N3 }\end{array}$ & $\begin{array}{c}\text { 6lu7 (2.16) } \\
\text { Complex with } \\
\text { N3 }\end{array}$ & $\begin{array}{c}\text { 6lu7 (2.16) } \\
\text { Complex with } \\
\text { N3 }\end{array}$ & $\begin{array}{c}\text { 5r82 (1.31) } \\
\text { Complex with } \\
\text { RZS }\end{array}$ & $\begin{array}{c}\text { 6yb7 (1.25) } \\
\text { Apo }\end{array}$ \\
\cline { 2 - 6 } & Rigid & Flex 41,145 & Flex 49,189 & Flex 49,189 & Flex 49,189 \\
\hline Beclabuvir & $\mathbf{- 1 0 . 4}$ & $\mathbf{- 1 0 . 0}$ & $\mathbf{- 1 0 . 5}$ & $\mathbf{- 8 . 5}$ & -8.1 \\
\hline Saquinavir & $\mathbf{- 9 . 3}$ & $\mathbf{- 9 . 2}$ & $\mathbf{- 8 . 9}$ & $\mathbf{- 9 . 2}$ & $\mathbf{- 8 . 3}$ \\
\hline Indinavir & $\mathbf{- 8 . 5}$ & -8.5 & -8.1 & -8.0 & $\mathbf{- 8 . 7}$ \\
\hline Nelfinavir & -8.0 & -7.8 & -8.3 & $-\mathbf{8 . 5}$ & -7.8 \\
\hline Amprenavir & -7.9 & -7.7 & -7.9 & -7.9 & -7.2 \\
\hline Tipranavir & -7.9 & -7.7 & $-\mathbf{8 . 8}$ & -8.0 & $\mathbf{- 8 . 2}$ \\
\hline Darunavir & -7.8 & $\mathbf{- 8 . 7}$ & -7.8 & -7.1 & -7.8 \\
\hline Ritonavir & -7.5 & -7.7 & -8.0 & -7.2 & -6.8 \\
\hline Lopinavir & -7.4 & -7.5 & -8.5 & $-\mathbf{8 . 5}$ & -7.6 \\
\hline Fosamprenavir & -7.3 & -8.1 & -8.0 & -8.4 & -7.3 \\
\hline Atazanavir & -7.2 & -7.2 & -7.5 & -8.0 & -7.5 \\
\hline
\end{tabular}


Table 2. Highest scoring approved drugs from SuperDrug2 database (kcal/mol). The target protease, Mpro of SARS-CoV-2, was rigid for Vina calculations and SMINA calculations were carried out with flexible residues at the active site. The resolution of the target structure is given in brackets $(\AA)$. The ligands (RZS or N3) were removed from the structure of the complex before docking with the ligand of interest.

\begin{tabular}{|l|c|c|c|c|c|}
\hline & Vina & SMINA & SMINA & SMINA & SMINA \\
\cline { 2 - 6 } & $\begin{array}{c}\text { 6lu7 (2.16) } \\
\text { Complex } \\
\text { with N3 }\end{array}$ & $\begin{array}{c}\text { 6lu7 (2.16) } \\
\text { Complex } \\
\text { with N3 }\end{array}$ & $\begin{array}{c}\text { 6lu7 (2.16) } \\
\text { Complex } \\
\text { with N3 }\end{array}$ & $\begin{array}{c}\text { 5r82 (1.31) } \\
\text { Complex } \\
\text { with RZS }\end{array}$ & $\begin{array}{c}\text { 6yb7 (1.25) } \\
\text { Apo }\end{array}$ \\
\cline { 2 - 6 } & Rigid & Flex 41,145 & Flex 49,189 & Flex 49,189 & Flex 49,189 \\
\hline Beclabuvir & $\mathbf{- 1 0 . 4}$ & $\mathbf{- 1 0 . 0}$ & $\mathbf{- 1 0 . 5}$ & -8.5 & -8.1 \\
\hline Nilotinib & $\mathbf{- 9 . 7}$ & -9.5 & -10.3 & -9.1 & -8.5 \\
\hline Lifitegrast & $\mathbf{- 9 . 4}$ & -9.4 & -8.8 & -9.2 & -8.5 \\
\hline Tadalafil & $\mathbf{- 9 . 4}$ & -9.2 & -9.9 & -9.4 & -8.2 \\
\hline Saquinavir & -9.3 & -9.2 & -8.9 & -9.2 & -8.3 \\
\hline Venetoclax & -9.3 & -9.4 & $\mathbf{- 1 0 . 6}$ & $\mathbf{- 9 . 7}$ & -9.0 \\
\hline Casopitant & -9.2 & -8.8 & -9.1 & -8.7 & -8.8 \\
\hline Digitoxin & -9.2 & $\mathbf{- 1 0 . 2}$ & $\mathbf{- 1 0 . 4}$ & -9.3 & $\mathbf{- 9 . 5}$ \\
\hline Digoxin & -9.2 & $\mathbf{- 1 0 . 2}$ & -10.0 & $\mathbf{- 9 . 5}$ & $\mathbf{- 9 . 6}$ \\
\hline Dihydroergocornine & -9.2 & -9.3 & -9.7 & $\mathbf{- 9 . 5}$ & -9.3 \\
\hline Ledipasvir & -9.2 & -8.4 & -9.5 & -8.7 & -9.1 \\
\hline Tirilazad & -9.2 & -9.8 & -10.1 & -9.4 & $\mathbf{- 9 . 6}$ \\
\hline Bisantrene & -9.1 & -8.6 & -9.0 & -9.1 & -8.9 \\
\hline Elbasvir & -9.1 & -8.8 & -9.2 & -8.3 & -8.1 \\
\hline Trametinib & -9.1 & -8.5 & -9.1 & -8.3 & -8.6 \\
\hline
\end{tabular}


Table 3. Estimated binding energies ( $\mathrm{kcal} / \mathrm{mol})$ and dissociation constants $(\mathrm{M})$, for docking of Main protease of SARS-CoV-2 with small molecule drugs in clinical trials for therapy of COVID-19. All drug categories in this Table, other than Direct Acting Antiviral (DAA) Protease Inhibitors (PI), represent potential off-target interactions.

\begin{tabular}{|c|c|c|c|c|c|c|}
\hline Potential Drug & Status & $\begin{array}{l}\text { Vina } \\
\text { score }\end{array}$ & $\begin{array}{l}\text { SMINA } \\
\text { score }\end{array}$ & $\begin{array}{c}\text { Dissociation } \\
\text { Constant }\end{array}$ & Category & $\begin{array}{l}\text { Approved for / } \\
\text { (Experimental) }\end{array}$ \\
\hline Danoprevir & Phase 4 & -9.3 & -10.0 & $1.5 \mathrm{E}-07$ & $\begin{array}{l}\text { DAA } \\
\text { PI }\end{array}$ & ( Hepatitis C ) \\
\hline Asc09f & Phase 3 & -8.1 & -8.0 & $1.1 \mathrm{E}-06$ & $\begin{array}{c}\text { DAA } \\
\text { PI }\end{array}$ & ( HIV/AIDS ) \\
\hline Darunavir & Phase 3 & -7.8 & -8.8 & $1.9 \mathrm{E}-06$ & $\begin{array}{c}\text { DAA } \\
\text { PI }\end{array}$ & HIV/AIDS \\
\hline Ritonavir & Phase 4 & -7.5 & -8.0 & $3.2 \mathrm{E}-06$ & $\begin{array}{l}\text { DAA } \\
\text { PI }\end{array}$ & HIV/AIDS \\
\hline Lopinavir & Phase 4 & -7.4 & -8.5 & 3.7E-06 & $\begin{array}{l}\text { DAA } \\
\text { PI }\end{array}$ & HIV/AIDS \\
\hline Sildenafil & Phase 3 & -8.2 & -8.4 & 9.7E-07 & & $\begin{array}{c}\text { Pulmonary arterial } \\
\text { hypertension }\end{array}$ \\
\hline Itraconazole & Phase 2 & -8.1 & -8.1 & $1.1 \mathrm{E}-06$ & & $\begin{array}{c}\text { Pulmonary } \\
\text { aspergillosis }\end{array}$ \\
\hline Sofosbuvir & Phase 3 & -8.0 & -8.4 & $1.4 \mathrm{E}-06$ & DAA & Hepatitis C \\
\hline Methylprednisolone & Phase 4 & -7.8 & -8.6 & 1.9E-06 & & $\begin{array}{c}\text { Rheumatism / } \\
\text { Allergy }\end{array}$ \\
\hline Baricitinib & Phase 3 & -7.5 & -7.9 & $3.2 \mathrm{E}-06$ & & $\begin{array}{c}\text { Rheumatoid } \\
\text { arthritis }\end{array}$ \\
\hline Remdesivir & Phase 3 & -7.5 & -7.8 & 3.2E-06 & DAA & $\begin{array}{l}\text { ( Ebola and } \\
\text { Marburg ) }\end{array}$ \\
\hline Ebastine & Phase 4 & -7.5 & -7.0 & 3.2E-06 & & Allergic rhinitis \\
\hline
\end{tabular}




\section{Figures}

Figure 1a : Comparison of experimentally determined binding mode of ligand N3 (6lu7) and the binding mode predicted with Vina. Experimentally determined ligand is green and predicted bound conformation of N3 is blue.

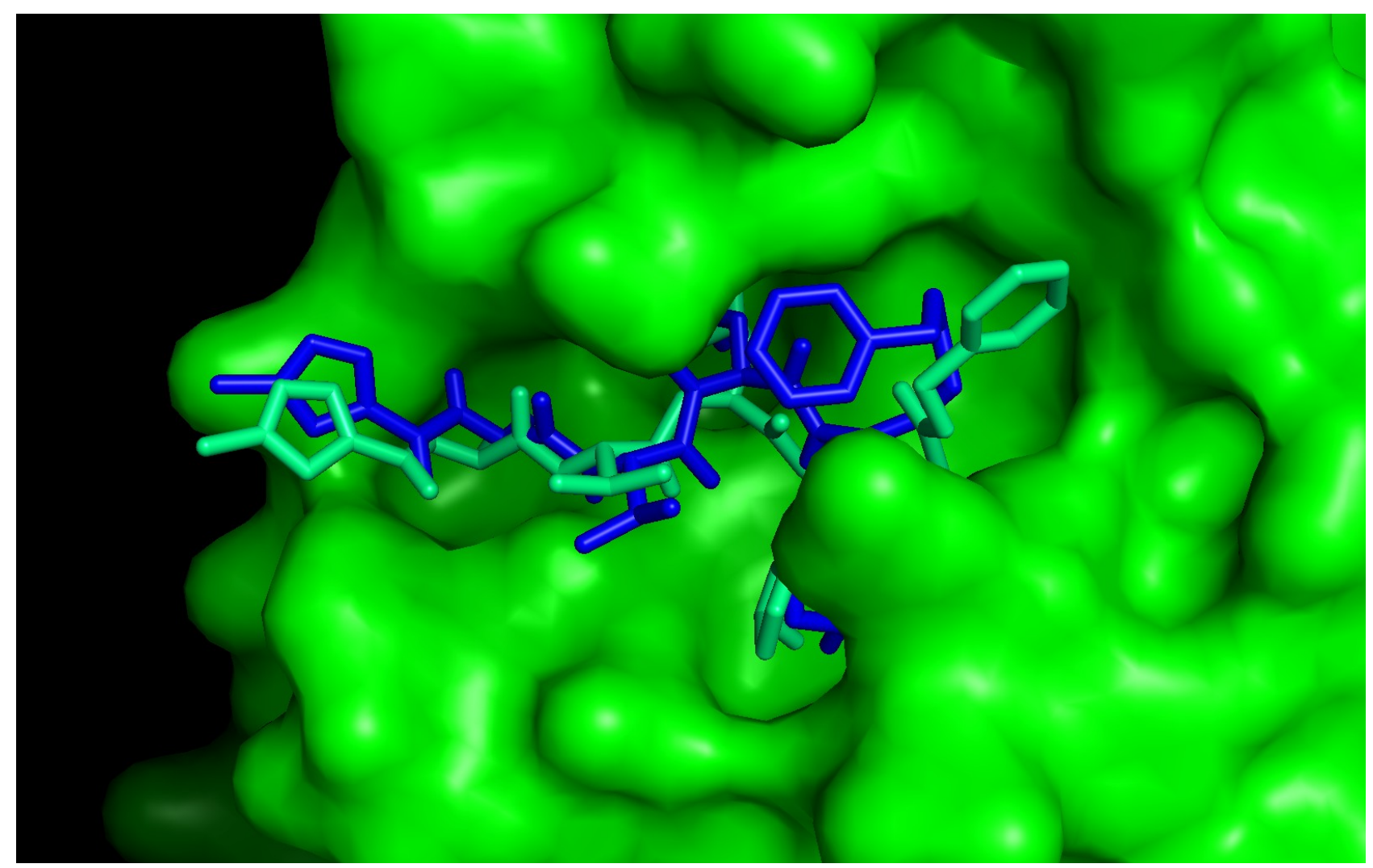


Figure 1b : Comparison of experimentally determined binding mode of ligand N3 (6lu7) and binding mode predicted with SMINA. Experimentally determined ligand is green and predicted bound conformation of N3 is blue.

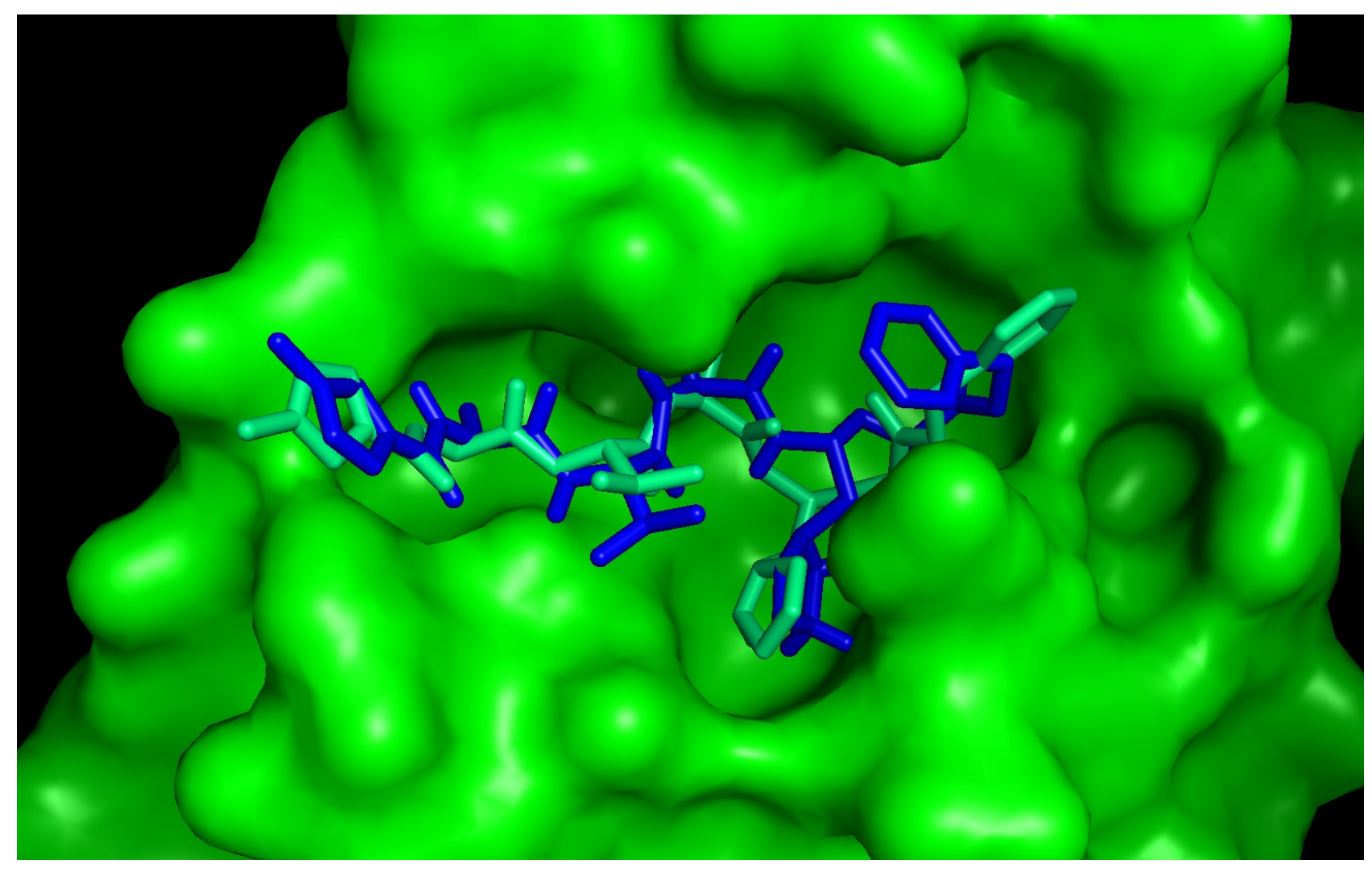


Figure 2 : Experimentally determined binding mode of ligand N3 (6lu7) and binding mode of Beclabuvir predicted with SMINA. Surface of target, SARS-CoV-2 Mpro, is green. N3 is green and Beclabuvir is blue.

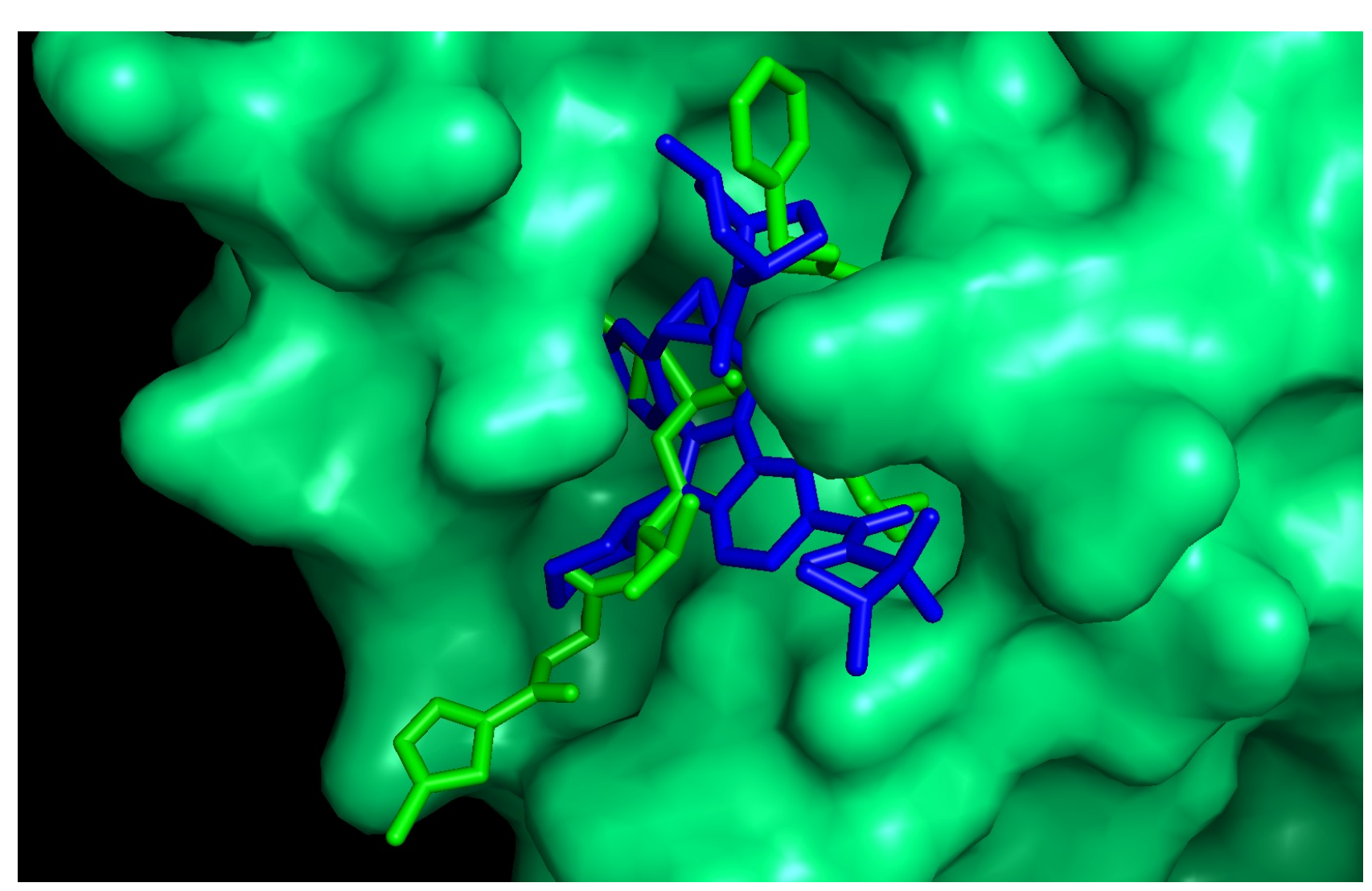


Figure 3 : Experimentally determined binding mode of ligand N3 (6lu7) and binding mode of Saquinavir predicted with SMINA. Surface of target, SARS-CoV-2 Mpro, is green. N3 is green and Saquinavir is blue.

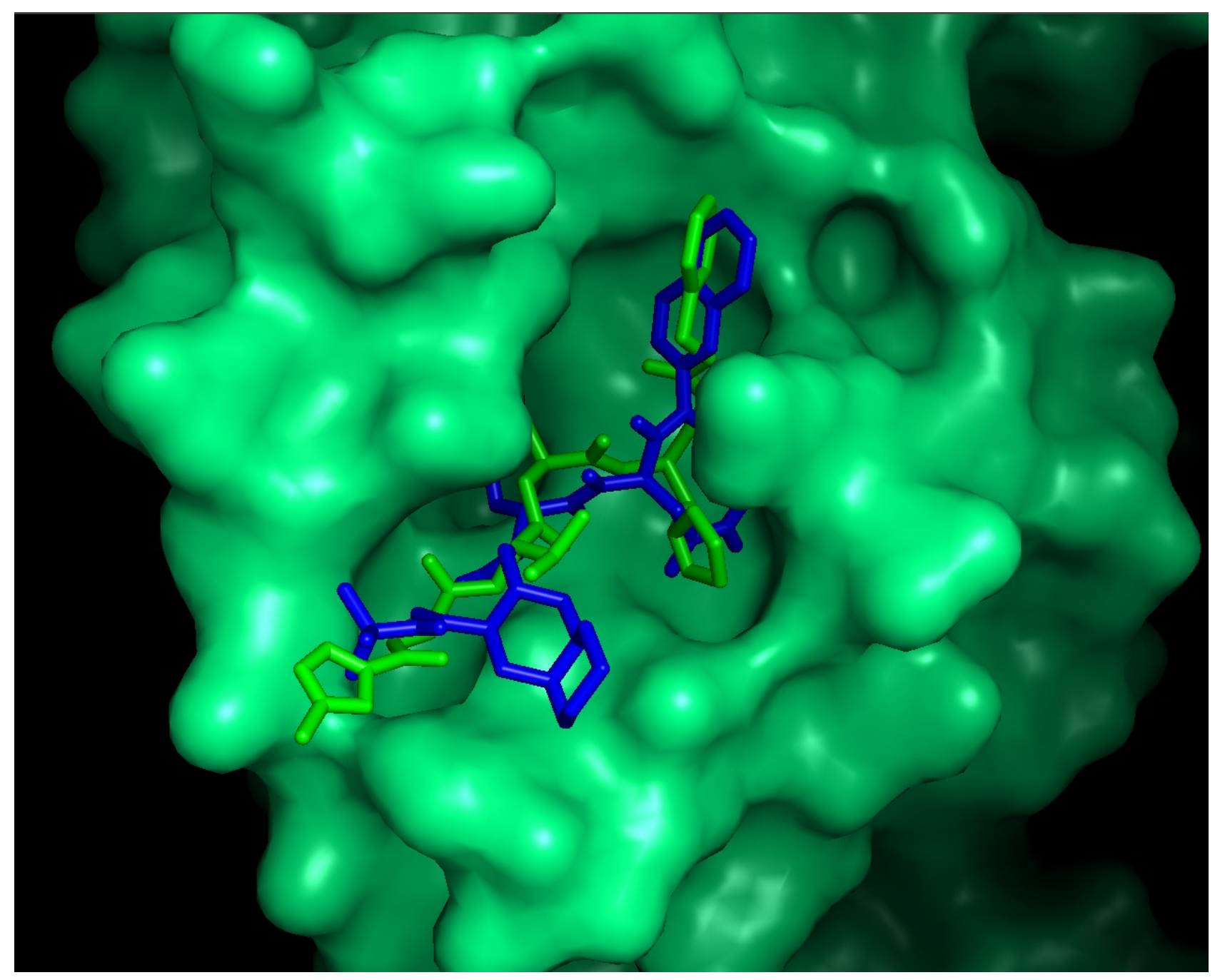




\section{References}

[1]Wu F et al. A new coronavirus associated with human respiratory disease in China. Nature 2020; 579(7798): 265-269.

[2]Zhao S et al. Preliminary estimation of the basic reproduction number of novel coronavirus (2019-nCoV) in China, from 2019 to 2020: A data-driven analysis in the early phase of the outbreak. International journal of infectious diseases 2020; 92: 214-217.

[3]Li G, De Clercq E. Therapeutic options for the 2019 novel coronavirus (2019-nCoV). Nature reviews. Drug discovery 2020; 19(3): 149-150.

[4]Lai CC, Shih TP, Ko WC, Tang HJ, Hsueh PR. Severe acute respiratory syndrome coronavirus 2 (SARS-CoV-2) and coronavirus disease-2019 (COVID-19): The epidemic and the challenges. International journal of antimicrobial agents 2020; 55(3): 105924-105924.

[5]Velavan TP, Meyer CG. The COVID-19 epidemic. Tropical medicine \& international health : TM \& IH 2020; 25(3): 278-280.

[6]ICTV. International Committee on Taxonomy of Viruses, Coronaviridae Study Group, The species Severe acute respiratory syndrome-related coronavirus: classifying 2019-nCoV and naming it SARS-CoV-2. Nature microbiology 2020; 5(4): 536-544. doi:10.1038/s41564-0200695-z

[7]Zhu N et al. A Novel Coronavirus from Patients with Pneumonia in China, 2019. The New England journal of medicine 2020; 382(8): 727-733.

[8]Zhou P et al. A pneumonia outbreak associated with a new coronavirus of probable bat origin. Nature 2020; 579(7798): 270-273. doi:10.1038/s41586-020-2012-7

[9]Lu H. Drug treatment options for the 2019-new coronavirus (2019-nCoV). Bioscience trends 2020; 14(1): 169-71. doi:10.5582/bst.2020.01020

[10]Fan H-H et al. Repurposing of clinically approved drugs for treatment of coronavirus disease 2019 in a 2019-novel coronavirus (2019-nCoV) related coronavirus model. Chinese medical journal 2020. doi:10.1097/CM9.0000000000000797

[11]Kruse RL. Therapeutic strategies in an outbreak scenario to treat the novel coronavirus originating in Wuhan, China. F1000Research 2020; 9: 72-72.

[12]Marshall GR. Computer-aided drug design. Annual review of pharmacology and toxicology 1987; 27: 193-213.

[13]Kuntz ID. Structure-based strategies for drug design and discovery. Science (New York, N.Y.) 1992; 257(5073): 1078-1082.

[14]Macalino SJY, Gosu V, Hong S, Choi S. Role of computer-aided drug design in modern drug discovery. Archives of pharmacal research 2015; 38(9): 1686-1701.

[15]Talluri S. Computational protein design of bacteriocins based on structural scaffold of 
aureocin A53. International Journal of Bioinformatics Research and Applications 2019; 15(2): 129-143. doi:10.1504/IJBRA.2019.099575

[16]Irwin JJ, Shoichet BK. Docking Screens for Novel Ligands Conferring New Biology. Journal of medicinal chemistry 2016; 59(9): 4103-4120.

[17]Mohanasundaram N, Sekhar T. Computational Studies of Molecular Targets Regarding the Adverse Effects of Isoniazid Drug for Tuberculosis. Current Pharmacogenomics and Personalized Medicine. 2018; 16(3): 210-218. doi:10.2174/1875692116666181108145230

[18]Strømgaard, K., Krogsgaard-Larsen, P. \& Madsen, U. (Eds.) 2017. Textbook of drug design and discovery. CRC press.

[19]Ashburn TT, Thor KB. Drug repositioning: identifying and developing new uses for existing drugs. Nature reviews. Drug discovery 2004; 3(8): 673-683.

[20]Xu J, Shi P-Y, Li H, Zhou J. Broad Spectrum Antiviral Agent Niclosamide and Its Therapeutic Potential. ACS infectious diseases 2020. doi:10.1021/acsinfecdis.0c00052

[21]Li H, Wang YM, Xu JY, Cao B. Potential antiviral therapeutics for 2019 Novel Coronavirus. Chinese journal of tuberculosis and respiratory diseases 2020; 43: E002-E002.

[22]Novac N. Challenges and opportunities of drug repositioning. Trends in pharmacological sciences 2013; 34(5): 267-272.

[23]Colson P, Rolain J-M, Lagier J-C, Brouqui P, Raoult D. Chloroquine and hydroxychloroquine as available weapons to fight COVID-19. International journal of antimicrobial agents 2020; 105932. doi:10.1016/j.ijantimicag.2020.105932

[24]Liu J et al. Hydroxychloroquine, a less toxic derivative of chloroquine, is effective in inhibiting SARS-CoV-2 infection in vitro. Cell Discovery. 2020; 6: 16.

[25]Wang $\mathrm{M}$ et al. Remdesivir and chloroquine effectively inhibit the recently emerged novel coronavirus (2019-nCoV) in vitro. Cell Research 2020; 30(3): 269-271. doi:10.1038/s41422020-0282-0

[26]Gao J, Tian Z, Yang X. Breakthrough: Chloroquine phosphate has shown apparent efficacy in treatment of COVID-19 associated pneumonia in clinical studies. Bioscience trends 2020; 14(1): 72-73. doi:10.5582/bst.2020.01047

[27]Gautret P et al. Hydroxychloroquine and azithromycin as a treatment of COVID-19: results of an open-label non-randomized clinical trial. International journal of antimicrobial agents 2020; 105949. doi:10.1016/j.ijantimicag.2020.105949

[28]Kupferschmidt K, Cohen J. Race to find COVID-19 treatments accelerates. Science 2020; 367(6485): 1412.

[29]Anand K, Ziebuhr J, Wadhwani P, Mesters JR, Hilgenfeld R. Coronavirus Main Proteinase (3CLpro) Structure: Basis for Design of Anti-SARS Drugs. Science 2003; 300(5626): 1763. 
[30]Zhang $L$ et al. $\alpha$-Ketoamides as Broad-Spectrum Inhibitors of Coronavirus and Enterovirus Replication: Structure-Based Design, Synthesis, and Activity Assessment. J. Med. Chem. 2020. doi:10.1021/acs.jmedchem.9b01828

[31]Liu S, Zheng Q, Wang Z. Potential covalent drugs targeting the main protease of the SARSCoV-2 coronavirus. Bioinformatics (Oxford, England) 2020.

doi:10.1093/bioinformatics/btaa224

[32]Tong TR. Severe Acute Respiratory Syndrome Coronavirus (SARS-CoV). Perspectives in Medical Virology 2006; 16: 43-95.

[33]Zhang L et al. Crystal structure of SARS-CoV-2 main protease provides a basis for design of improved \$-ketoamide inhibitors. Science (New York, N.Y.) 2020. doi:10.1126/science.abb3405

[34]Wang Z, Chen X, Lu Y, Chen F, Zhang W. Clinical characteristics and therapeutic procedure for four cases with 2019 novel coronavirus pneumonia receiving combined Chinese and Western medicine treatment. Biosci Trends 2020; 14(1): 64-68.

[35]Cao B et al. A Trial of Lopinavir-Ritonavir in Adults Hospitalized with Severe Covid-19. The New England journal of medicine 2020. doi:10.1056/NEJMoa2001282

[36]Arun K G et al. Structure-based Design, Synthesis and Biological Evaluation of Peptidomimetic SARS-CoV 3CLpro Inhibitors. Bioorg Med Chem Lett. 2007; 17(21): 58765880 .

[37]Wu C et al. Analysis of therapeutic targets for SARS-CoV-2 and discovery of potential drugs by computational methods. Acta Pharmaceutica Sinica B 2020. doi:10.1016/j.apsb.2020.02.008

[38]Zhang $L$ et al. Crystal structure of SARS-CoV-2 main protease provides a basis for design of improved $\alpha$-ketoamide inhibitors. Science 2020. doi:10.1126/science.abb3405

[39]Jin Z et al. Structure-based drug design, virtual screening and high-throughput screening rapidly identify antiviral leads targeting COVID-19. bioRxiv 2020. doi:10.1101/2020.02.26.964882

[40]Zhang D-H, Wu K-L, Zhang X, Deng S-Q, Peng B. In silico screening of Chinese herbal medicines with the potential to directly inhibit 2019 novel coronavirus. Journal of integrative medicine 2020; 18(2): 152-158. doi:10.1016/j.joim.2020.02.005

[41]Ton A-T, Gentile F, Hsing M, Ban F, Cherkasov A. Rapid Identification of Potential Inhibitors of SARS-CoV-2 Main Protease by Deep Docking of 1.3 Billion Compounds. Molecular informatics 2020. doi:10.1002/minf.202000028

[42]Burley SK, Berman HM, Kleywegt GJ, Markley JL, Nakamura H, Velankar S. Protein Data Bank (PDB): The Single Global Macromolecular Structure Archive. Methods in molecular biology (Clifton, N.J.) 2017; 1607: 627-641.

[43]Liu X, Zhang B, Jin Z, Yang H, Rao Z. The crystal structure of 2019-nCoV main protease in complex with an inhibitor N3. PDB ID 6LU7 2020. 
[44]O'Boyle NM, Banck M, James CA, Morley C, Vandermeersch T, Hutchison GR. Open Babel: An open chemical toolbox. Journal of cheminformatics 2011; 3: 33.

[45]Hanwell MD, Curtis DE, Lonie DC, Vandermeersch T, Zurek E, Hutchison GR. Avogadro: an advanced semantic chemical editor, visualization, and analysis platform. Journal of Cheminformatics 2012; 4(1): 17.

[46]Sayle RA, Milner-White EJ. RASMOL: biomolecular graphics for all. Trends in biochemical sciences 1995; 20(9): 374.

[47]Zhang Y, Skolnick J. TM-align: a protein structure alignment algorithm based on the TMscore. Nucleic Acids Res 2005; 33(7): 2302-2309.

[48]Siramshetty VB et al. SuperDRUG2: a one stop resource for approved/marketed drugs. Nucleic acids research 2018; 46(D1): D1137-D1143.

[49]Goede A, Dunkel M, Mester N, Frommel C, Preissner R. SuperDrug: a conformational drug database. Bioinformatics (Oxford, England) 2005; 21(9): 1751-1753.

[50]Hähnke VD, Kim S, Bolton EE. PubChem chemical structure standardization. Journal of cheminformatics 2018; 10(1): 36-76.

[51]Wishart DS et al. DrugBank 5.0: a major update to the DrugBank database for 2018. Nucleic acids research 2018; 46(D1): D1074-D1082.

[52]Sterling T, Irwin JJ. ZINC 15 - Ligand Discovery for Everyone. J. Chem. Inf. Model. 2015; 55(11): 2324-2337. doi:10.1021/acs.jcim.5b00559

[53]Trott O, Olson AJ. AutoDock Vina: improving the speed and accuracy of docking with a new scoring function, efficient optimization, and multithreading. Journal of computational chemistry 2010; 31(2): 455-461.

[54]Koes DR, Baumgartner MP, Camacho CJ. Lessons learned in empirical scoring with smina from the CSAR 2011 benchmarking exercise. Journal of chemical information and modeling 2013; 53(8): 1893-1904.

[55]Pagadala NS, Syed K, Tuszynski J. Software for molecular docking: a review. Biophysical reviews 2017; 9(2): 91-102.

[56]Jeon S et al. Identification of antiviral drug candidates against SARS-CoV-2 from FDAapproved drugs. bioRxiv. 2020; -. doi:10.1101/2020.03.20.999730

[57]Ko M et al. Screening of FDA-approved drugs using a MERS-CoV clinical isolate from South Korea identifies potential therapeutic options for COVID-19. bioRxiv 2020. doi:10.1101/2020.02.25.965582

[58]Guo T et al. Cardiovascular Implications of Fatal Outcomes of Patients With Coronavirus Disease 2019 (COVID-19). JAMA Cardiol 2020.

[59]Tan J et al. pH-dependent Conformational Flexibility of the SARS-CoV Main Proteinase (Mpro) Dimer: Molecular Dynamics Simulations and Multiple X-ray Structure Analyses. Journal of Molecular Biology 2005; 354(1): 25-40. 
[60]Leach AR, Shoichet BK, Peishoff CE. Prediction of protein-ligand interactions. Docking and scoring: successes and gaps. Journal of medicinal chemistry 2006; 49(20): 5851-5855.

[61]Wang Z et al. Comprehensive evaluation of ten docking programs on a diverse set of proteinligand complexes: the prediction accuracy of sampling power and scoring power. Physical chemistry chemical physics 2016; 18(18): 12964-12975.

[62]Gaillard T. Evaluation of AutoDock and AutoDock Vina on the CASF-2013 Benchmark. Journal of chemical information and modeling 2018; 58(8): 1697-1706.

[63]Masters L, Eagon S, Heying M. Evaluation of consensus scoring methods for AutoDock Vina, smina and idock. Journal of molecular graphics \& modelling 2020; 96: 107532107532.

[64]Rismanbaf A. Potential Treatments for COVID-19; a Narrative Literature Review. Archives of Academic Emergency Medicine 2020; 8(1): .

[65]Kontoyianni M. Docking and Virtual Screening in Drug Discovery. Methods in molecular biology 2017; 1647: 255-266.

[66]Li J, Fu A, Zhang L. An Overview of Scoring Functions Used for Protein-Ligand Interactions in Molecular Docking. Interdisciplinary sciences, computational life sciences 2019; 11(2): 320-328.

[67]Sarma P, Prajapat M, Avti P, Kaur H, Kumar S, Medhi B. Therapeutic options for the treatment of 2019-novel coronavirus: An evidence-based approach. Indian journal of pharmacology 2020; 52(1): 1-5.

[68]Markham A, Keam SJ. Danoprevir: First Global Approval. Drugs 2018; 78(12): 1271-1276.

[69]Garimella T et al. Effects of a Fixed-Dose Co-Formulation of Daclatasvir, Asunaprevir, and Beclabuvir on the Pharmacokinetics of a Cocktail of Cytochrome P450 and Drug Transporter Substrates in Healthy Subjects. Drugs in R\&D 2018; 18(1): 55-65.

[70]Chen C et al. Thalidomide Combined with Low-dose Glucocorticoid in the Treatment of COVID-19 Pneumonia. Preprints 2020; 202002.0395.v1.

[71]Mehta P, McAuley DF, Brown M, Sanchez E, Tattersall RS, Manson JJ, =HLH Across Speciality Collaboration U. COVID-19: consider cytokine storm syndromes and immunosuppression. Lancet (London, England) 2020; 395(10229): 1033-1034.

[72]Yi Y, Lagniton PN, Ye S, Li E, Xu R-H. COVID-19: what has been learned and to be learned about the novel coronavirus disease. International journal of biological sciences 2020; 16(10): 1753-1766.

[73]Shang L, Zhao J, Hu Y, Du R, Cao B. On the use of corticosteroids for 2019-nCoV pneumonia. The Lancet 2020; 395(10225): 683-684. doi:10.1016/S0140-6736(20)30361-5

[74]Stebbing J, Phelan A, Griffin I, Tucker C, Oechsle O, Smith D, Richardson P. COVID-19: combining antiviral and anti-inflammatory treatments. The Lancet. Infectious diseases 2020; 20(4): 400-402. 
[75]Baden LR, Rubin EJ. Covid-19 -- The Search for Effective Therapy. N Engl J Med 2020. doi:10.1056/NEJMe2005477

[76]Luca P, Giulio R. Molecular Docking: Shifting Paradigms in Drug Discovery. International Journal of Molecular Sciences. 2019; 20: 4331.

[77]Zheng Y-Y, Ma Y-T, Zhang J-Y, Xie X. COVID-19 and the cardiovascular system. Nature Reviews Cardiology 2020. 
Appendix-1

SMINA scores for docking of drugs in clinical trials with Main protease of SARS-CoV-2.

\begin{tabular}{|c|c|c|c|c|}
\hline & $\begin{array}{c}\text { 6lu7 (2.16) } \\
\text { Complex } \\
\text { (N3) }\end{array}$ & \begin{tabular}{|c|} 
6lu7 (2.16) \\
Complex \\
(N3)
\end{tabular} & $\begin{array}{c}\text { 5r82 (1.31) } \\
\text { Complex } \\
(\mathrm{RZS})\end{array}$ & $\begin{array}{c}\text { 6yb7 (1.25) } \\
\text { Apo }\end{array}$ \\
\hline & Flex 41,145 & Flex 49,189 & Flex 49,189 & Flex 49,189 \\
\hline Danoprevir & -8.9 & -10.0 & -8.4 & -8.2 \\
\hline Asc09f & -8.0 & -7.3 & -7.5 & -7.5 \\
\hline Darunavir & -8.8 & -7.8 & -7.1 & -7.8 \\
\hline Ritonavir & -7.8 & -8.0 & -7.2 & -6.8 \\
\hline Lopinavir & -7.5 & -8.5 & -8.5 & -7.6 \\
\hline Sildenafil & -8.2 & -8.4 & -8.2 & -7.5 \\
\hline Itraconazole & -8.0 & -8.1 & -7.7 & -7.1 \\
\hline Sofosbuvir & -8.0 & -8.4 & -8.4 & -7.7 \\
\hline Methylprednisolone & -7.6 & -8.6 & -7.6 & -8.2 \\
\hline Baricitinib & -7.2 & -7.9 & -7.4 & -7.3 \\
\hline Remdesivir & -7.8 & -7.1 & -7.5 & -7.1 \\
\hline Ebastine & -6.8 & -6.7 & -7.0 & -6.5 \\
\hline
\end{tabular}

